\title{
BOUNDARY VALUE PROBLEMS FOR BAGLEY-TORVIK FRACTIONAL DIFFERENTIAL EQUATIONS AT RESONANCE
}

\author{
SVATOSLAV STANĚK
}

Received 15 October, 2015

\begin{abstract}
We investigate the nonlocal fractional boundary value problem $u^{\prime \prime}=A^{c} D^{\alpha} u+$ $f\left(t, u,{ }^{c} D^{\mu} u, u^{\prime}\right), u^{\prime}(0)=u^{\prime}(T), \Lambda(u)=0$, at resonance. Here, $\alpha \in(1,2), \mu \in(0,1), f$ and $\Lambda: C[0, T] \rightarrow \mathbb{R}$ are continuous. We introduce a "three-component" operator $\delta$ which first component is related to the fractional differential equation and remaining ones to the boundary conditions. Solutions of the problem are given by fixed points of $\delta$. The existence of fixed points of $\checkmark$ is proved by the Leray-Schauder degree method.
\end{abstract}

2010 Mathematics Subject Classification: 34A08; 26A33; 34B15

Keywords: fractional differential equation, boundary value problem at resonance, Caputo fractional derivative, Leray-Schauder degree, maximum principle

\section{INTRODUCTION}

Let $T>0$ be given and $J=[0, T]$. Denote by $\mathcal{A}$ the set of (generally nonlinear) functionals $\Lambda: C(J) \rightarrow \mathbb{R}$ which are

(a) continuous, $\Lambda(0)=0$,

(b) increasing, that is, $x, y \in C(J), x(t)<y(t)$ on $J \Rightarrow \Lambda(x)<\Lambda(y)$.

Remark 1. Let $\Lambda \in \mathcal{A}$ be linear. Then it follows from property (b) of $\Lambda$ that $\Lambda$ takes bounded sets into bounded sets. Hence $\Lambda$ is a linear bounded functional.

Example 1. Let $p \in C(J)$ be positive, $n \in \mathbb{N}, 0 \leq t_{0}<t_{1}<\cdots<t_{n} \leq T$, and $a_{k}>0, k=0,1, \ldots, n$. Then the functionals

$$
\begin{aligned}
& \Lambda_{1}(x)=\max \{x(t): t \in J\}, \Lambda_{2}(x)=\min \{x(t): t \in J\}, \\
& \Lambda_{3}(x)=\int_{0}^{T} p(s)(x(s))^{2 n-1} \mathrm{~d} s, \Lambda_{4}(x)=\sum_{k=0}^{n} a_{k} x\left(t_{k}\right)
\end{aligned}
$$

and their linear combinations with positive coefficients belong to the set $\mathcal{A}$.

Supported by the grant No. 14-06958S of the Grant Agency of the Czech Republic. 
We discuss the fractional boundary value problem

$$
\begin{gathered}
u^{\prime \prime}(t)=A^{c} D^{\alpha} u(t)+f\left(t, u(t),{ }^{c} D^{\mu} u(t), u^{\prime}(t)\right), \\
u^{\prime}(0)=u^{\prime}(T), \quad \Lambda(u)=0, \quad \Lambda \in \mathcal{A} .
\end{gathered}
$$

Here, ${ }^{c} D$ denotes the Caputo fractional derivative, $A \in \mathbb{R}, \alpha \in(1,2), \mu \in(0,1)$, and the function $f$ satisfies the condition:

(H) there exists $\Delta>0$ such that $f \in C(\mathscr{D} \times[-\Delta, \Delta])$, where

$$
\mathscr{D}=J \times[-\Delta T, \Delta T] \times[-\Delta K, \Delta K]), \quad K=\frac{T^{1-\mu}}{\Gamma(2-\mu)},
$$

and

$$
f(t, x, y,-\Delta) \leq 0, \quad f(t, x, y, \Delta) \geq 0 \quad \text { for }(t, x, y) \in \mathscr{D} .
$$

Equation 1.1 is the fractional differential equation of the Bagley-Torvik type. Its special case is the equation $u^{\prime \prime}=A^{c} D^{3 / 2} u+a u+\varphi(t)$. This equation with ${ }^{c} D^{3 / 2}$ replaced by the Riemann-Liouville fractional derivative $D^{3 / 2}$ is called the BagleyTorvik equation. Torvik and Bagley [22] used this equation in modelling the motion of a rigid plate immersing in a Newtonian fluid. Analytical and numerical solutions of the problem

$$
u^{\prime \prime}=A D^{3 / 2} u+a u+\varphi(t), u(0)=0, u^{\prime}(0)=0,
$$

are given in $[13,16,18]$, while for the problem

$$
u^{\prime \prime}=A^{c} D^{\alpha} u+a u+\varphi(t), u(0)=u_{0}, u^{\prime}(0)=u_{1},
$$

in $[5,6,8,11,23]$. The existence results for solutions of the generalized Bagley-Torvik equation (1.1) satisfying the boundary conditions $u^{\prime}(0)=0, u(T)+a u^{\prime}(T)=0$ are given in [20]. Here, $f$ is a Carathéodory function.

Definition 1. We say that $u \in C^{2}(J)$ is a solution of problem (1.1), (1.2) if $u$ satisfies the boundary conditions (1.2) and (1.1) holds for $t \in J$.

We recall that the Riemann-Liouville fractional integral $I^{\gamma}$ of order $\gamma>0$ of a function $x: J \rightarrow \mathbb{R}$ is defined as $[10,13,16]$

$$
I^{\gamma} x(t)=\int_{0}^{t} \frac{(t-s)^{\gamma-1}}{\Gamma(\gamma)} x(s) \mathrm{d} s,
$$

and the Caputo fractional derivative ${ }^{c} D^{\gamma} x$ of order $\gamma>0, \gamma \notin \mathbb{N}$, of a function $x: J \rightarrow \mathbb{R}$ is given by the formula $[10,13]$

$$
{ }^{c} D^{\gamma} x(t)=\frac{\mathrm{d}^{n}}{\mathrm{~d} t^{n}} \int_{0}^{t} \frac{(t-s)^{n-\gamma-1}}{\Gamma(n-\gamma)}\left(x(s)-\sum_{k=0}^{n-1} \frac{x^{(k)}(0)}{k !} s^{k}\right) \mathrm{d} s,
$$


where $n=[\gamma]+1,[\gamma]$ means the integral part of $\gamma$ and $\Gamma$ is the Euler gamma function. If $x \in C^{n}(J)$ and $n-1<\gamma<n$, then

$$
{ }^{c} D^{\gamma} x(t)=\int_{0}^{t} \frac{(t-s)^{n-\gamma-1}}{\Gamma(n-\gamma)} x^{(n)}(s) \mathrm{d} s=I^{n-\gamma} x^{(n)}(t) .
$$

In particular, if $x \in C^{2}(J)$ and $\alpha \in(1,2), \mu \in(0,1)$, then

$$
\begin{gathered}
{ }^{c} D^{\alpha} x(t)=\int_{0}^{t} \frac{(t-s)^{1-\alpha}}{\Gamma(2-\alpha)} x^{\prime \prime}(s) \mathrm{d} s, t \in J, \\
{ }^{c} D^{\alpha} x(t)=\frac{\mathrm{d}}{\mathrm{d} t} \int_{0}^{t} \frac{(t-s)^{1-\alpha}}{\Gamma(2-\alpha)}\left(x^{\prime}(s)-x^{\prime}(0)\right) \mathrm{d} s={ }^{c} D^{\alpha-1} x^{\prime}(t), \quad t \in J, \\
{ }^{c} D^{\mu} x(t)=\frac{\mathrm{d}}{\mathrm{d} t} \int_{0}^{t} \frac{(t-s)^{-\mu}}{\Gamma(1-\mu)}(x(s)-x(0)) \mathrm{d} s=I^{1-\mu} x^{\prime}(t), t \in J .
\end{gathered}
$$

It is well known [10,13] that $I^{\gamma}: C(J) \rightarrow C(J)$ for $\gamma \in(0,1)$. Therefore, if $x \in$ $C^{2}(J)$, then ${ }^{c} D^{\alpha} x,{ }^{c} D^{\mu} x \in C(J)$ for $\alpha \in(1,2)$ and $\mu \in(0,1)$.

We will show that problem $(1.1),(1.2)$ is at resonance. The linear function $x(t)=$ $a t+b$ is a solution of the problem $u^{\prime \prime}-A^{c} D^{\alpha} u=0, u^{\prime}(0)=u^{\prime}(T)$, for each $a, b \in \mathbb{R}$. Let us consider the set of all functions $a t+b$ which are solutions of the equation $\Lambda(a t+b)=0$, where $\Lambda$ is from (1.2).

If $\Lambda$ is linear, then $b=-\frac{a \Lambda(t)}{\Lambda(1)}$. Hence $\left\{a\left(t-\frac{\Lambda(t)}{\Lambda(1)}\right): a \in \mathbb{R}\right\}$ is the set of solutions to problem $u^{\prime \prime}-A^{c} D^{\alpha} u=0,(1.2)$. This set is a one-dimensional linear subspace of $C^{2}(J)$.

Let $\Lambda$ be nonlinear. If $a=0$, then $b=0$. Let $a \in \mathbb{R} \backslash\{0\}$. By our Lemma 1 (for $\lambda=1$ ), there exists $\xi_{a} \in J$ such that $a \xi_{a}+b=0$. Hence $b=-a \xi_{a}$ and the equality $\Lambda\left(a\left(t-\xi_{a}\right)\right)=0$ is true. $\xi_{a}$ is determined uniquely. If this is not true, then there exists $\rho_{a} \in J, \rho_{a} \neq \xi_{a}$, such that $\Lambda\left(a\left(t-\rho_{a}\right)\right)=0$. Since $a\left(t-\xi_{a}\right) \neq a\left(t-\rho_{a}\right)$ for all $t \in J$, and therefore either $a\left(t-\xi_{a}\right)<a\left(t-\rho_{a}\right)$ or $a\left(t-\xi_{a}\right)>a\left(t-\rho_{a}\right)$ on $J$, it follows from property (b) of $\Lambda$ that $\Lambda\left(a\left(t-\xi_{a}\right)\right) \neq \Lambda\left(a\left(t-\rho_{a}\right)\right)$, which is impossible. Consequently, $u=0$ and $\left\{a\left(t-\xi_{a}\right): a \in \mathbb{R} \backslash\{0\}\right\}$ is the set of solutions to the problem $u^{\prime \prime}-A^{c} D^{\alpha} u=0,(1.2)$. In contrast to previous case, this set is not a one-dimensional linear subspace of $C^{2}(J)$.

In order to show the solvability of problem (1.1),(1.2), we have to overcome troubles that derivatives are of fractional order, the problem is at resonance and finally that $\Lambda$ in the boundary conditions (1.2) is generally a nonlinear functional. To this end, an auxiliary "three-component" operator $\delta$ is introduced. Its first component is related to equation (1.1) and remaining ones to the boundary conditions (1.2). Solutions of (1.1), (1.2) are given by fixed points of $\delta$. The existence of fixed points of $\delta$ is proved by means of the Leray-Schauder degree method [7].

In the literature, see $[1-4,12,14,19]$ and references therein, existence results for fractional boundary value problems at resonance are usually proved by using the the coincidence degree theory due to Mawhin [15]. 
Our main result is as follows.

Theorem 1. Let $(H)$ hold and let $A>0$. Then problem (1.1), (1.2) has at least one solution.

The paper is organized as follows. In Section 2 we state the results which are used in the next sections. Section 3 is devoted to auxiliary boundary value problems. To this end operators $\mathcal{Q}, \mathcal{S}, \mathcal{K}_{\lambda}$ and $\mathscr{H}_{\lambda}$ are introduced and their properties are given. In Section 4 Theorem 1 is proved. An example demonstrates our results.

Throughout the paper $\alpha \in(1,2), \mu \in(0,1), K=\frac{T^{1-\mu}}{\Gamma(2-\mu)}$ and $\|x\|=\max \{|x(t)|: t \in$ $J\}$ is the norm in $C(J)$.

\section{PRELIMINARIES}

This section contains the results that we will need in the next sections.

Lemma 1. Let $\Lambda \in \mathcal{A}$ and let the equality

$$
\Lambda(x)+(\lambda-1) \Lambda(-x)=0
$$

hold for some $x \in C(J)$ and $\lambda \in[0,1]$. Then there exists $\xi \in J$ such that $x(\xi)=0$.

Proof. Assume that the statement is not true. Then either $x>0$ or $x<0$ on $J$. If $x>0$ on $J$, then $\Lambda(x)>0, \Lambda(-x)<0$, and therefore $\Lambda(x)+(\lambda-1) \Lambda(-x)>0$, which is impossible. Similarly, $x<0$ on $J$ leads to a contradiction.

The following maximal principle follows immediately from [17, Lemma 2.1] and [9, Lemma 2.7] and its proof.

Lemma 2 (Maximum principle). Let $t_{0} \in(0, T], x \in C^{1}\left[0, t_{0}\right], x(t) \leq x\left(t_{0}\right)$ for $t \in\left[0, t_{0}\right], x(0)<x\left(t_{0}\right)$ and $x^{\prime}\left(t_{0}\right)=0$. Let $\gamma \in(0,1)$. Then

$$
\left.{ }^{c} D^{\gamma} x(t)\right|_{t=t_{0}}>0 \text {. }
$$

Corollary 1. Let $t_{0} \in(0, T], x \in C^{1}\left[0, t_{0}\right], x(t) \geq x\left(t_{0}\right)$ for $t \in\left[0, t_{0}\right], x(0)>x\left(t_{0}\right)$ and $x^{\prime}\left(t_{0}\right)=0$. Let $\gamma \in(0,1)$. Then

$$
\left.{ }^{c} D^{\gamma} x(t)\right|_{t=t_{0}}<0 .
$$

Lemma 3 ([21]). Let $r \in C(J)$ and $\gamma \in(0,1)$. Then the initial value problem

$$
x^{\prime}(t)=A^{c} D^{\gamma} x(t)+r(t), x(0)=a, \quad A, a \in \mathbb{R},
$$

has the unique solution

$$
x(t)=a+\int_{0}^{t} r(s) \mathrm{d} s+A \int_{0}^{t}\left(\int_{0}^{s}(s-\xi)^{-\gamma} E_{1-\gamma, 1-\gamma}\left(A(s-\xi)^{1-\gamma}\right) r(\xi) \mathrm{d} \xi\right) \mathrm{d} s,
$$

where

$$
E_{1-\gamma, 1-\gamma}(z)=\sum_{k=0}^{\infty} \frac{z^{k}}{\Gamma((k+1)(1-\gamma))}, \quad z \in \mathbb{R},
$$

is the classical Mittag-Leffler function. 
Lemma 4 ([24, Lemma 2.2]). Let $\rho \in(0,1)$ and let $E_{\rho, \rho}$ be the Mittag-Leffler function. Then

$$
E_{\rho, \rho}(z)>0, \quad E_{\rho, \rho}^{\prime}(z)>0 \quad \text { for } z \in \mathbb{R} .
$$

We also need the following result.

Lemma 5. Let $h \in C(J)$ and $A, c_{1}, c_{2} \in \mathbb{R}$. Then the initial value problem

$$
u^{\prime \prime}(t)=A^{c} D^{\alpha} u(t)+h(t), u(0)=c_{2}, u^{\prime}(0)=c_{1},
$$

has the unique solution

$$
\begin{aligned}
u(t)= & c_{1} t+c_{2}+\int_{0}^{t}(t-s) h(s) \mathrm{d} s \\
& +A \int_{0}^{t}(t-s)\left(\int_{0}^{s}(s-\xi)^{1-\alpha} E_{2-\alpha, 2-\alpha}\left(A(s-\xi)^{2-\alpha}\right) h(\xi) \mathrm{d} \xi\right) \mathrm{d} s .
\end{aligned}
$$

Proof. Since ${ }^{c} D^{\alpha} x(t)={ }^{c} D^{\alpha-1} x^{\prime}(t)$ for $t \in J$ and $x \in C^{2}(J)$, the equation of (2.1) can be written as

$$
u^{\prime \prime}(t)=A^{c} D^{\alpha-1} u^{\prime}(t)+h(t) .
$$

Hence, by Lemma 3 (for $r=h$ and with $x$ and $\gamma$ replaced by $u^{\prime}$ and $\alpha-1$ ),

$$
\begin{aligned}
u^{\prime}(t)= & c_{1}+\int_{0}^{t} h(s) \mathrm{d} s \\
& +A \int_{0}^{t}\left(\int_{0}^{s}(s-\xi)^{1-\alpha} E_{2-\alpha, 2-\alpha}\left(A(s-\xi)^{2-\alpha}\right) h(\xi) \mathrm{d} \xi\right) \mathrm{d} s,
\end{aligned}
$$

where $u^{\prime}(0)=c_{1}$. Consequently, $u(t)=c_{2}+\int_{0}^{t} u^{\prime}(s) \mathrm{d} s$ is the unique solution of problem (2.1) and (2.2) follows.

\section{OPERATORS}

In this section auxiliary operators are introduced and their properties are proved. The most important of these operators is an operator $\delta$ by which the solvability of problem (1.1), (1.2) is proved in Section 4.

Let

$$
\chi_{1}(x)=\left\{\begin{array}{ll}
\Delta T & \text { for } x>\Delta T, \\
x & \text { for }|x| \leq \Delta T, \\
-\Delta T & \text { for } x<-\Delta T,
\end{array} \quad \chi_{2}(y)= \begin{cases}\Delta K & \text { for } y>\Delta K, \\
y & \text { for }|y| \leq \Delta K, \\
-\Delta K & \text { for } y<-\Delta K,\end{cases}\right.
$$

where $\Delta$ and $K$ are from $(H)$. Let

$$
\tilde{f}(t, x, y, z)=f\left(t, \chi_{1}(x), \chi_{2}(y), z\right) \quad \text { for }(t, x, y, z) \in J \times \mathbb{R}^{2} \times[-\Delta, \Delta]
$$


and

$$
f^{*}(t, x, y, z)= \begin{cases}\tilde{f}(t, x, y, \Delta)+\frac{z-\Delta}{z} & \text { if } z>\Delta, \\ \tilde{f}(t, x, y, z) & \text { if }|z| \leq \Delta, \\ \tilde{f}(t, x, y,-\Delta)-\frac{z+\Delta}{z} & \text { if } z<-\Delta .\end{cases}
$$

Under condition $(H), f^{*} \in C\left(J \times \mathbb{R}^{3}\right)$,

$$
\left.\begin{array}{cl}
f^{*}(t, x, y,-\Delta) \leq 0, & f^{*}(t, x, y, \Delta) \geq 0 \quad \text { for }(t, x, y) \in J \times \mathbb{R}^{2}, \\
f^{*}(t, x, y, z)<0 & \text { for }(t, x, y, z) \in J \times \mathbb{R}^{2} \times(-\infty,-\Delta), \\
f^{*}(t, x, y, z)>0 & \text { for }(t, x, y, z) \in J \times \mathbb{R}^{2} \times(\Delta, \infty),
\end{array}\right\}
$$

and

$$
\left|f^{*}(t, x, y, z)\right| \leq E \quad \text { for }(t, x, y, z) \in J \times \mathbb{R}^{3},
$$

where

$$
E=1+\max \{|f(t, x, y, z)|:(t, x, y) \in \mathscr{D}, z \in[-\Delta, \Delta]\} .
$$

Consider the fractional differential equation

$$
u^{\prime \prime}(t)=A^{c} D^{\alpha} u(t)+f^{*}\left(t, u(t),{ }^{c} D^{\mu} u(t), u^{\prime}(t)\right)
$$

associated to equation (1.1). Keeping in mind Lemma 5 define operators $\mathbb{Q}: C^{1}(J) \rightarrow$ $C(J)$ and $8: C^{1}(J) \times \mathbb{R}^{2} \rightarrow C^{1}(J) \times \mathbb{R}^{2}$ by the formulae

$$
\begin{aligned}
(\mathcal{Q} x)(t)= & f^{*}\left(t, x(t),{ }^{c} D^{\mu} x(t), x^{\prime}(t)\right) \\
& +A \int_{0}^{t}(t-s)^{1-\alpha} E_{2-\alpha, 2-\alpha}\left(A(t-s)^{2-\alpha}\right) f^{*}\left(s, x(s),{ }^{c} D^{\mu} x(t), x^{\prime}(s)\right) \mathrm{d} s, \\
\delta\left(x, c_{1}, c_{2}\right) & =\left(c_{1} t+c_{2}+\int_{0}^{t}(t-s)(\mathcal{Q} x)(s) \mathrm{d} s, c_{1}+\int_{0}^{T}(\mathcal{Q} x)(s) \mathrm{d} s, c_{2}+\Lambda(x)\right),
\end{aligned}
$$

where $\Lambda$ is from (1.2).

Lemma 6. Let $(H)$ hold. If $\left(x, c_{1}, c_{2}\right)$ is a fixed point of the operator 8 , then $x$ is a solution of problem (3.3), (1.2) and $x^{\prime}(0)=c_{1}, x(0)=c_{2}$.

Proof. Let $\left(x, c_{1}, c_{2}\right)$ be a fixed point of the operator 8 , that is, $8\left(x, c_{1}, c_{2}\right)=$ $\left(x, c_{1}, c_{2}\right)$. Then

$$
\begin{gathered}
x(t)=c_{1} t+c_{2}+\int_{0}^{t}(t-s)(\mathcal{Q} x)(s) \mathrm{d} s, \quad t \in J, \\
\int_{0}^{T}(\mathcal{Q} x)(s) \mathrm{d} s=0, \\
\Lambda(x)=0 .
\end{gathered}
$$


It follows from (3.4) and Lemma 5 (for $h(t)=f^{*}\left(t, x(t),{ }^{c} D^{\mu} x(t), x^{\prime}(t)\right)$ ) that $x(0)=c_{2}, x^{\prime}(0)=c_{1}$ and $x$ is a solution of (3.3).

Since (cf. (3.4))

$$
x^{\prime}(t)=c_{1}+\int_{0}^{t}(Q 2 x)(s) \mathrm{d} s, \quad t \in J,
$$

we conclude from (3.5) that $x^{\prime}(T)=c_{1}$. Hence $x^{\prime}(0)=x^{\prime}(T)$. The last equality together with (3.6) give that $x$ satisfies the boundary conditions (1.2). Consequently, $x$ is a solution of problem (3.3), (1.2) and $x^{\prime}(0)=c_{1}, x(0)=c_{2}$.

In order to prove that the operator $\delta$ admits a fixed point, for $\lambda \in[0,1]$, we first introduce an operator $\mathcal{K}_{\lambda}: C^{1}(J) \times \mathbb{R}^{2} \rightarrow C^{1}(J) \times \mathbb{R}^{2}$,

$$
\begin{aligned}
& \mathcal{K}_{\lambda}\left(x, c_{1}, c_{2}\right) \\
& =\left(c_{1} t+c_{2}, c_{1}+(1-\lambda) x^{\prime}(0)+\lambda \int_{0}^{T}(\mathcal{Q} x)(s) \mathrm{d} s, c_{2}+\Lambda(x)+(\lambda-1) \Lambda(-x)\right) .
\end{aligned}
$$

Let

$$
\begin{aligned}
& \Omega=\left\{\left(x, c_{1}, c_{2}\right) \in C^{1}(J) \times \mathbb{R}^{2}\right. \\
&\left.:\|x\|<\Delta T+1,\left\|x^{\prime}\right\|<\Delta+1,\left|c_{1}\right|<\Delta+1,\left|c_{2}\right|<\Delta T+1\right\},
\end{aligned}
$$

where $\Delta$ is from $(H)$.

Lemma 7. Let $(H)$ hold and let $A>0$. Then

$$
\operatorname{deg}\left(\mathfrak{d}-\mathcal{K}_{1}, \Omega, 0\right) \neq 0
$$

where "deg" stands for the Leray-Schauder degree and $d$ is the identity operator on $C^{1}(J) \times \mathbb{R}^{2}$.

Proof. Let $M:[0,1] \times C^{1}(J) \times \mathbb{R} \rightarrow C^{1}(J) \times \mathbb{R}, M\left(\lambda, x, c_{1}, c_{2}\right)=\mathcal{K}_{\lambda}\left(x, c_{1}, c_{2}\right)$. Since $f^{*} \in C\left(J \times \mathbb{R}^{3}\right)$, we conclude from Lemma 4 that $Q$ is a continuous operator. As $\Lambda$ is continuous and takes bounded sets into bounded sets, it is easy to prove that $M$ is a completely continuous operator.

Due to

$$
\mathcal{K}_{0}\left(-x,-c_{1},-c_{2}\right)=-\mathcal{K}_{0}\left(x, c_{1}, c_{2}\right) \quad \text { for }\left(x, c_{1}, c_{2}\right) \in C^{1}(J) \times \mathbb{R}^{2},
$$

$\mathcal{K}_{0}$ is an odd operator.

Assume that $M\left(\lambda_{0}, x, c_{1}, c_{2}\right)=\left(x, c_{1}, c_{2}\right)$ for some $\left(x, c_{1}, c_{2}\right) \in C^{1}(J) \times \mathbb{R}^{2}$ and $\lambda_{0} \in[0,1]$. Then

$$
\begin{gathered}
x(t)=c_{1} t+c_{2}, \quad t \in J, \\
\left(1-\lambda_{0}\right) x^{\prime}(0)+\lambda_{0} \int_{0}^{T}(\mathcal{Q} x)(s) \mathrm{d} s=0, \\
\Lambda(x)+\left(\lambda_{0}-1\right) \Lambda(-x)=0 .
\end{gathered}
$$


Lemma 1 together with (3.11) give $x(\xi)=0$ for some $\xi \in J$. Hence (cf. (3.9)) $c_{1} \xi+c_{2}=0$, and therefore $x(t)=c_{1}(t-\xi)$ on $J$.

We now prove that

$$
\left|c_{1}\right| \leq \Delta .
$$

Let $c_{1}>\Delta$. Then $x^{\prime}=c_{1}>\Delta$ on $J$, and therefore $f^{*}\left(t, x(t),{ }^{c} D^{\mu} x(t), x^{\prime}(t)\right)>0$ for $t \in J$ by (3.1). This fact together with $A>0$ and Lemma 4 imply $(Q x)(t)>0$ on $J$. Hence $\left(1-\lambda_{0}\right) c_{1}+\lambda_{0} \int_{0}^{T}(Q x)(s) \mathrm{d} s>0$, which contradicts (3.10). Therefore $c_{1} \leq \Delta$. Similarly, if $c_{1}<-\Delta$, then we have $f^{*}\left(t, x(t),{ }^{c} D^{\mu} x(t), x^{\prime}(t)\right)<0$ and $(Q x)(t)<0$ for $t \in J$, which again contradicts (3.10). Hence (3.12) is true.

Consequently, $|x(t)|=\left|c_{1}(t-\xi)\right| \leq \Delta T,\left|x^{\prime}(t)\right|=\left|c_{1}\right| \leq \Delta,\left|{ }^{c} D^{\mu} x(t)\right|=$ $\left|I^{1-\mu} x^{\prime}(t)\right| \leq \Delta K$ on $J$ and $\left|c_{2}\right|=|x(0)| \leq \Delta T$. As a result,

$$
M\left(\lambda, x, c_{1}, c_{2}\right) \neq\left(x, c_{1}, c_{2}\right) \quad \text { for }\left(x, c_{1}, c_{2}\right) \in \partial \Omega \text { and } \lambda \in[0,1] .
$$

Hence, by the Borsuk antipodal theorem and the homotopy property, the relations

$$
\begin{gathered}
\operatorname{deg}\left(d-\mathcal{K}_{0}, \Omega, 0\right) \neq 0, \\
\operatorname{deg}\left(\downarrow-\mathcal{K}_{0}, \Omega, 0\right)=\operatorname{deg}\left(d-\mathcal{K}_{1}, \Omega, 0\right)
\end{gathered}
$$

hold. Combining these relations we obtain (3.8).

Finally, let for $\lambda \in[0,1]$ an operator $\mathscr{H}_{\lambda}: C^{1}(J) \times \mathbb{R}^{2} \rightarrow C^{1}(J) \times \mathbb{R}^{2}$ be defined as

$$
\mathscr{H}_{\lambda}\left(x, c_{1}, c_{2}\right)=\left(c_{1} t+c_{2}+\lambda \int_{0}^{t}(t-s)(\mathcal{Q} x)(s) \mathrm{d} s, c_{1}+\int_{0}^{T}(\mathcal{Q} x)(s) \mathrm{d} s, c_{2}+\Lambda(x)\right) .
$$

Then, for $\left(x, c_{1}, c_{2}\right) \in C^{1}(J) \times \mathbb{R}^{2}$,

$$
\begin{gathered}
\mathscr{H}_{0}\left(x, c_{1}, c_{2}\right)=\mathcal{K}_{1}\left(x, c_{1}, c_{2}\right), \\
\mathscr{H}_{1}\left(x, c_{1}, c_{2}\right)=\delta\left(x, c_{1}, c_{2}\right) .
\end{gathered}
$$

Lemma 8. Let $(H)$ hold. Let $V:[0,1] \times C^{1}(J) \times \mathbb{R} \rightarrow C^{1}(J) \times \mathbb{R}$ and $V\left(\lambda, x, c_{1}, c_{2}\right)=\mathscr{H}_{\lambda}\left(x, c_{1}, c_{2}\right)$. Then $V$ is a completely continuous operator.

Proof. We first prove that $V$ is continuous. To this end let $\left\{x_{n}\right\} \subset C^{1}(J),\left\{c_{n, i}\right\} \subset$ $\mathbb{R}, i=1,2,\left\{\lambda_{n}\right\} \subset[0,1]$ be convergent sequences and let $\lim _{n \rightarrow \infty} x_{n}=x$ in $C^{1}(J)$, $\lim _{n \rightarrow \infty} c_{n, i}=c_{i}, \lim _{n \rightarrow \infty} \lambda_{n}=\lambda$ in $\mathbb{R}$, where $x \in C^{1}(J), c_{i}, \lambda \in \mathbb{R}, i=1,2$. Then $\lim _{n \rightarrow \infty} f^{*}\left(t, x_{n}(t),{ }^{c} D^{\mu} x_{n}(t), x_{n}^{\prime}(t)\right)=f^{*}\left(t, x(t),{ }^{c} D^{\mu} x(t), x^{\prime}(t)\right)$ uniformly on $J$. This together with Lemma 4 imply that $\lim _{n \rightarrow \infty}\left(\mathcal{Q} x_{n}\right)(t)=(\mathcal{Q} x)(t)$ uniformly on $J$. Hence

$$
\begin{gathered}
\lim _{n \rightarrow \infty}\left(c_{n, 1} t+c_{n, 2}+\lambda_{n} \int_{0}^{t}(t-s)\left(\mathcal{Q} x_{n}\right)(s) \mathrm{d} s\right)=c_{1} t+c_{2}+\lambda \int_{0}^{t}(t-s)(\mathcal{Q} x)(s) \mathrm{d} s, \\
\lim _{n \rightarrow \infty}\left(c_{n, 1}+\lambda_{n} \int_{0}^{t}\left(\mathcal{Q} x_{n}\right)(s) \mathrm{d} s\right)=c_{1}+\lambda \int_{0}^{t}(\mathcal{Q} x)(s) \mathrm{d} s
\end{gathered}
$$


uniformly on $J$. Besides,

$$
\begin{aligned}
\lim _{n \rightarrow \infty}\left(c_{n, 1}+\int_{0}^{T}\left(\mathcal{Q} x_{n}\right)(s) \mathrm{d} s\right) & =c_{1}+\int_{0}^{T}(\mathcal{Q} x)(s) \mathrm{d} s, \\
\lim _{n \rightarrow \infty}\left(c_{n, 2}+\Lambda\left(x_{n}\right)\right) & =c_{2}+\Lambda(x) .
\end{aligned}
$$

Consequently, $V$ is a continuous operator.

Let $\Phi \subset C^{1}(J) \times \mathbb{R}^{2}$ be bounded and let $\|x\| \leq L,\left\|x^{\prime}\right\| \leq L,\left|c_{1}\right| \leq L,\left|c_{2}\right| \leq L$ for $\left(x, c_{1}, c_{2}\right) \in \Phi$, where $L$ is a positive constant. Let $W=E_{2-\alpha, 2-\alpha}\left(|A| T^{2-\alpha}\right)$. Then, by (3.2) and Lemma 4, the relation

$$
\begin{aligned}
|(Q \mathcal{Q} x)(t)| & \leq E+|A| E \int_{0}^{t}(t-s)^{1-\alpha} E_{2-\alpha, 2-\alpha}\left(A(t-s)^{2-\alpha}\right) \mathrm{d} s \\
& \leq E+|A| E W \int_{0}^{t}(t-s)^{1-\alpha} \mathrm{d} s \leq E+|A| E W \frac{T^{2-\alpha}}{2-\alpha}=H
\end{aligned}
$$

holds for $t \in J$ and $\left(x, c_{1}, c_{2}\right) \in \Phi$. Hence

$$
\begin{aligned}
& \left|c_{1} t+c_{2}+\lambda \int_{0}^{t}(t-s)(\mathcal{Q} x)(s) \mathrm{d} s\right| \leq L(T+1)+\frac{H T^{2}}{2}, \\
& \left|c_{1}+\lambda \int_{0}^{T}(\mathcal{Q} x)(s) \mathrm{d} s\right| \leq L+H T, \\
& \left|c_{2}+\Lambda(x)\right| \leq L+\max \{|\Lambda(-L)|, \Lambda(L)\}
\end{aligned}
$$

for $t \in J,\left(x, c_{1}, c_{2}\right) \in \Phi$ and $\lambda \in[0,1]$, and therefore the set $V([0,1] \times \Phi)=\left\{V\left(\lambda, x, c_{1}, c_{2}\right): \lambda \in\right.$ $\left.[0,1],\left(x, c_{1}, c_{2}\right) \in \Phi\right\}$ is bounded in $C^{1}(J) \times \mathbb{R}^{2}$. In view of $\|\mathcal{Q} x\| \leq H$ we see that the set $\left\{c_{1}+\lambda \int_{0}^{t}(\mathcal{Q} x)(s) \mathrm{d} s:\left(x, c_{1}, c_{2}\right) \in \Phi, \lambda \in[0,1]\right\}$ is equicontinuous on $J$.

Hence the Arzelà-Ascoli theorem and the Bolzano-Weierstrass compactness theorem in $\mathbb{R}$ guarantee that the set $V([0,1] \times \Phi)$ is relatively compact in $C^{1}(J) \times \mathbb{R}^{2}$. Consequently, $V$ is completely continuous.

\section{THE PROOF OF THEOREM 1 AND AN EXAMPLE}

Proof. Suppose that $\left(x, c_{1}, c_{2}\right) \in C^{1}(J) \times \mathbb{R}^{2}$ is a fixed point of $\mathscr{H}_{\lambda}$ for some $\lambda \in[0,1]$, that is, $\mathscr{H}_{\lambda}\left(x, c_{1}, c_{2}\right)=\left(x, c_{1}, c_{2}\right)$. If $\lambda=0$, then it follows from the proof of Lemma 7 (cf. (3.13)) that $\left(x, c_{1}, c_{2}\right) \in \Omega$, where $\Omega$ is given in (3.7). Let $\lambda \in(0,1]$. Then

$$
\begin{gathered}
x(t)=c_{1} t+c_{2}+\lambda \int_{0}^{t}(t-s)(\mathcal{Q} x)(s) \mathrm{d} s, \quad t \in J, \\
\int_{0}^{T}(\mathcal{Q} x)(s) \mathrm{d} s=0, \\
\Lambda(x)=0 .
\end{gathered}
$$


Hence

$$
x^{\prime}(t)=c_{1}+\lambda \int_{0}^{t}(Q 2 x)(s) \mathrm{d} s, \quad t \in J,
$$

so $x^{\prime}(0)=c_{1}$, and, by (4.2), $x^{\prime}(T)=c_{1}+\lambda \int_{0}^{T}(\mathcal{Q} x)(s) \mathrm{d} s=c_{1}$. Consequently,

$$
x^{\prime}(0)=x^{\prime}(T) \text {. }
$$

Suppose that $c_{1}>\Delta$, where $\Delta$ is from $(H)$. Then $f^{*}\left(0, x(0), 0, c_{1}\right)>0$ by (3.1), and therefore $f^{*}\left(t, x(t),{ }^{c} D^{\mu} x(t), x^{\prime}(t)\right)>0$ on a right neighbourhood of $t=0$. If there is some $\xi \in(0, T]$ such that $f^{*}\left(t, x(t),{ }^{c} D^{\mu} x(t), x^{\prime}(t)\right)>0$ on $[0, \xi)$ and $f^{*}\left(\xi, x(\xi),\left.{ }^{c} D^{\mu} x(t)\right|_{t=\xi}, x^{\prime}(\xi)\right)=0$, then $(Q 2 x)(t)>0$ on $[0, \xi]$ because $A>0$, which gives $x^{\prime}(t)>c_{1}$ for $t \in(0, \xi]$. Hence $f^{*}\left(t, x(t),{ }^{c} D^{\mu} x(t), x^{\prime}(t)\right)>0$ on $[0, \xi]$, contrary to $f^{*}\left(\xi, x(\xi),\left.{ }^{c} D^{\mu} x(t)\right|_{t=\xi}, x^{\prime}(\xi)\right)=0$. Consequently,

$$
f^{*}\left(t, x(t),{ }^{c} D^{\mu} x(t), x^{\prime}(t)\right)>0, \quad(Q x)(t)>0, \quad t \in J .
$$

Thus $x^{\prime}(T)>c_{1}=x^{\prime}(0)$, which contradicts (4.5). Hence $c_{1} \leq \Delta$. Similarly, we can prove that $c_{1} \geq-\Delta$. To summarize, $\left|c_{1}\right| \leq \Delta$.

Suppose that $\max \left\{x^{\prime}(t): t \in J\right\}=x^{\prime}(\xi)>\Delta$. Then $\xi \in(0, T)$ and $x^{\prime}(\xi)-x^{\prime}(0)>$ 0 . By (4.4), $x \in C^{2}(J)$ and $x^{\prime \prime}=\lambda Q \mathcal{Q} x$. Hence $x^{\prime \prime}(\xi)=0$ and by Lemma 5 and (2.3) (for $\left.h(t)=\lambda f^{*}\left(t, x(t),{ }^{c} D^{\mu} x(t), x^{\prime}(t)\right)\right)$ the equality

$$
x^{\prime \prime}(t)=A^{c} D^{\alpha-1} x^{\prime}(t)+\lambda f^{*}\left(t, x(t),{ }^{c} D^{\mu} x(t), x^{\prime}(t)\right), t \in J,
$$

holds. Lemma 2 (for $t_{0}=\xi, \gamma=\alpha-1$ and $x$ replaced by $x^{\prime}$ ) shows that $\left.{ }^{c} D^{\alpha-1} x^{\prime}(t)\right|_{t=\xi}>0$. Hence

$$
x^{\prime \prime}(\xi)=\left.A^{c} D^{\alpha-1} x^{\prime}(t)\right|_{t=\xi}+\lambda f^{*}\left(\xi, x(\xi),\left.{ }^{c} D^{\mu} x(t)\right|_{t=\xi}, x^{\prime}(\xi)\right)>0,
$$

which is impossible. Hence $x^{\prime}(t) \leq \Delta$ for $t \in J$. Similarly, by Corollary 1 , we can prove that $x^{\prime} \geq-\Delta$ on $J$. Consequently,

$$
\left|x^{\prime}(t)\right| \leq \Delta, t \in J
$$

Next, it follows from (4.3) and Lemma 1 that $x(\tau)=0$ for some $\tau \in J$. Therefore $|x(t)|=\left|\int_{\tau}^{t} x^{\prime}(s) \mathrm{d} s\right| \leq \Delta|t-\tau| \leq \Delta T,\left|{ }^{c} D^{\mu} x(t)\right|=\left|I^{1-\mu} x^{\prime}(t)\right| \leq \Delta K$. As $c_{1}=$ $x^{\prime}(0)$ and $c_{2}=x(0)$, we have proved

$$
\|x\| \leq \Delta T,\left\|{ }^{c} D^{\mu} x\right\| \leq \Delta K,\left\|x^{\prime}\right\| \leq \Delta,\left|c_{1}\right| \leq \Delta T,\left|c_{2}\right| \leq \Delta,
$$

which implies $V\left(\lambda, x, c_{1}, c_{2}\right) \neq\left(x, c_{1}, c_{2}\right)$ for $\left(x, c_{1}, c_{2}\right) \in \partial \Omega$ and $\lambda \in[0,1]$, where $V$ is from Lemma 8. Combinig Lemma 8 with the homotopy property we have

$$
\operatorname{deg}\left(\mathcal{l}-\mathscr{H}_{0}, \Omega, 0\right)=\operatorname{deg}\left(\mathcal{l}-\mathscr{H}_{1}, \Omega, 0\right) .
$$

This equality together with (3.8) and (3.13) give

$$
\operatorname{deg}\left(d-\mathscr{H}_{1}, \Omega, 0\right) \neq 0 \text {. }
$$


Hence there exists a fixed point $\left(x, c_{1}, c_{2}\right)$ of $\mathscr{H}_{1}$. Lemma 6 and (3.14) guarantee that $x$ is a fixed point of problem (3.3), (1.2) and $c_{1}=x^{\prime}(0), c_{2}=x(0)$. Due to (4.6), $f^{*}\left(t, x(t),{ }^{c} D^{\mu} x(t), x^{\prime}(t)\right)=f\left(t, x(t),{ }^{c} D^{\mu} x(t), x^{\prime}(t)\right)$ for $t \in J$, and therefore $x$ is a solution of problem (1.1), (1.2).

Example 2. Let $p \in C\left(J \times \mathbb{R}^{3}\right)$ be bounded, $a, b, c \in C(J), c>0$ on $J$, and $n \in \mathbb{N}$, $\beta, \gamma \in(0,2 n-1)$. Then the function

$$
f(t, x, y, z)=p(t, x, y, z)+a(t)|x|^{\beta-1} x+b(t)|y|^{\gamma}+c(t) z^{2 n-1}
$$

satisfies condition $(H)$. Really, let $|p(t, x, y, z)| \leq L$ for $(t, x, y, z) \in J \times \mathbb{R}^{3}$ and $c_{*}=\min \{c(t): t \in J\}$. Since

$$
\lim _{v \rightarrow \infty}\left(L v^{1-2 n}+\|a\| T^{\beta} v^{\beta+1-2 n}+\|b\| K^{\gamma} v^{\gamma+1-2 n}\right)=0, \quad K=\frac{T^{1-\mu}}{\Gamma(2-\mu)},
$$

there exists $\Delta>0$ such that

$$
L \Delta^{1-2 n}+\|a\| T^{\beta} \Delta^{\beta+1-2 n}+\|b\| K^{\gamma} \Delta^{\gamma+1-2 n} \leq c_{*} .
$$

Hence $L+\|a\|(\Delta T)^{\beta}+\|b\|(\Delta K)^{\gamma} \leq c_{*} \Delta^{2 n-1}$, and therefore for $(t, x, y) \in \mathscr{D}$, where $\mathscr{D}$ is from $(H)$, the inequalities

$$
\begin{aligned}
f(t, x, y, \Delta) & \geq-L-\|a\|(\Delta T)^{\beta}-\|b\|(\Delta K)^{\gamma}+c_{*} \Delta^{2 n-1} \geq 0, \\
f(t, x, y,-\Delta) & \leq L+\|a\|(\Delta T)^{\beta}+\|b\|(\Delta K)^{\gamma}-c_{*} \Delta^{2 n-1} \leq 0
\end{aligned}
$$

hold. Theorem 1 gives that the equation

$$
\begin{aligned}
u^{\prime \prime}= & A^{c} D^{\alpha} u+p\left(t, u,{ }^{c} D^{\mu} u, u^{\prime}\right) \\
& +a(t)|u|^{\beta-1} u+\left.\left.b(t)\right|^{c} D^{\mu} u\right|^{\gamma}+c(t)\left(u^{\prime}\right)^{2 n-1}, \quad A>0,
\end{aligned}
$$

has at least one solution $u$ satisfying the boundary conditions (1.2) and $\|u\| \leq \Delta T$, $\left\|{ }^{c} D^{\mu} u\right\| \leq \Delta K,\left\|u^{\prime}\right\| \leq \Delta$.

In particular, there exists a solution of (4.7) satisfying the boundary conditions

$$
\min \{u(t): t \in J\}=0, u^{\prime}(0)=u^{\prime}(T),
$$

that is, $u$ is a nonnegative solution of the problem.

\section{REFERENCES}

[1] C. Bai, "Existence result for boundary value problem of nonlinear impulsive fractional differential equation at resonance," J. Appl. Math. Comput., vol. 39, pp. 421-443, 2012.

[2] Z. Bai, "On solutions of some fractional $m$-point boundary value problems at resonance," Electron. J. Qual. The. Differ. Equ., vol. 37, pp. 1-15, 2010.

[3] Z. Bai, "Solvability for a class of fractional $m$-point boundary value problem at resonance," Comput. Math. Appl., vol. 62, pp. 1192-1302, 2011.

[4] T. Chen, W. Liu, and Z. Hu, "A boundary value problem for fractional differential equation with p-laplacian operator at resonance," Nonlinear Anal., vol. 75, pp. 3210-3217, 2012.

[5] V. Daftardar-Gejji and H. Jafari, "Adomian decomposition: a tool for solving a system of fractional differential equations," J. Math. Anal. Appl., vol. 301, pp. 508-518, 2005. 
[6] Y. Cenesiz, Y. Keskin, and A. Kurnaz, "The solution of the bagley-torvik equation with the generalized taylor collocation method,” J. Franklin Institute, vol. 347, pp. 452-466, 2010.

[7] K. Deimling, Nonlinear Functional Analysis. Springer, Berlin, 1985.

[8] K. Deimling and N. J. Ford, "Numerical solution of the bagley-torvik equation," BIT, vol. 42, no. 3, pp. 490-507, 2002.

[9] Z. Denton and A. S. Vatsala, "Nonotone iterative technique for finite systems of nonlinear riemann-liouville fractional differential equations," Opuscula Math., vol. 31, no. 3, pp. 327-339, 2011.

[10] K. Diethelm, The Analysis of Fractional Differential Equations, ser. Lectures Notes in Mathematics. Springer, Berlin-Heidelberg, 2010.

[11] J. T. Edwards, N. J. Ford, and A. C. Simpson, "The numerical solution of linear multi-term fractional differential equations: systems of equations," J. Comput. Appl. Math., vol. 148, pp. 401418, 2002.

[12] Z. Hu, W. Liu, and T. Chen, "Two-point boundary value problems for fractional differential equations at resonace," Bull. Malays. Math. Sci. Soc., vol. 36, no. 3, pp. 747-755, 2013.

[13] A. A. Kilbas, H. M. Srivastava, and J. J. Trujillo, Theory and Applications of Fractional Differential Equations. Elsevier B.V., Amsterdam, 2006.

[14] N. Kosmatov, "A boundary value problem of fractional order at resonance," Electron. J. Differ. Equ., vol. 2010, no. 135, pp. 1-10, 2010.

[15] J. Mawhin, Topological Degree Method in Nonlinear Boundary Value Problems, ser. CBMS. Amer. Math. Soc., Providence, R. I., 1979, vol. 40.

[16] I. Podlubny, Fractional Differential Equations, ser. Mathematics in Science and Engineering. Academic Press, San Diego, 1999, vol. 198.

[17] J. D. Ramirez and A. S. Vatsala, "Generalized monotone iterative technique for caputo fractional differential equation with periodic boundary condition via initial value problem," International $J$. Diff. Equ., vol. 2012, no. ID 842813, pp. 1-17, 2012, doi: 10.1155/2012/842813.

[18] S. Saha Ray and R. K. Bera, "Analytical solution of the bagley torvik equation by adomian decomposition method," Appl. Math. Comp., vol. 168, pp. 398-410, 2005.

[19] T. Shen, W. Liu, T. Chen, and X. Shen, "Solvability of fractional multi-point boundary-value problems with $p$-laplacian operator at resonance," Electron. J. Differ. Equ., vol. 2014, no. 58, pp. $1-10,2014$.

[20] S. Staněk, "Two-point boundary value problems for the generalized bagley-torvik fractional differential equation," Cent. Eur. J. Math., vol. 11, no. 3, pp. 574-593, 2013.

[21] S. Staněk, "Periodic problem for the generalized basset fractional differential equation," Fract. Calc. Appl. Anal., vol. 18, no. 5, pp. 1277-1290, 2015, doi: 10.1515/fca-2015-0073.

[22] P. J. Torvik and R. L. Bagley, "On the appearance of the fractional derivative in the behavior of real materials," ASME J. Appl. Mech., vol. 51, pp. 294-298, 1984.

[23] Z. H. Wang and X. Wang, "General solution of the bagley-torvik equation with fractional-order derivative," Commun. Nonlinear Sci. Numer. Simul., vol. 15, pp. 1279-1285, 2010.

[24] Z. Wei, Q. Li, and J. Che, "Initial boundary value problems for fractional differential equations involving a riemann-liouville sequential fractional derivative," J. Math. Anal. Appl., vol. 367, pp. 260-272, 2010.

Author's address

Svatoslav Staněk

Department of Mathematical Analysis, Faculty of Science

Current address: Palacký University, 17. listopadu 12, 77146 Olomouc, Czech Republic

E-mail address: svatoslav.stanek@upol.cz 